\title{
NB-IoT Pervasive Communications for Renewable Energy Source Monitoring
}

\author{
Farooque Hassan Kumbhar \\ Department of Computer Science \\ National University of Computer and Emerging Science \\ Pakistan
}

\begin{abstract}
Renewable sources like solar and wind energy have seen a drastic increase in the market, especially in developing countries where electricity prices are high and QoS and QoE, both are at their lowest. In this paper, we innovate by proposing a paradigm of smart off-grid from sensing using an Internet of Things (IoT) based smart meter for continuous monitoring, to reporting a daily user on their smart devices using IoT middleware. Our proposed smart off-grid system keeps track of the performance and faults of the off-grid equipment. Under communication technology scrutiny, we model 3GPP Narrow Band IoT (NB-IoT) collision and success probability of grouping smart meter communications to avoid random access channel (RACH) congestion. The proposed smart off-grid communications outperform existing systems and achieve 1.3 to 20 times higher SINR, more than 30 Mbps data rate in 4G, three times higher data rate in NB-IoT, 25\% fewer collisions and 25\% higher success rate.
\end{abstract}

Keywords-NB-IoT; smart off-grid; RACH; $4 G$ LTE

\section{INTRODUCTION}

Low cost and reliable energy sources have always been and will always be a major part of human interest. Users and investors are moving towards off-grid solutions like nuclear, wind and solar, powering 12-volt appliances from a bulb to an air-conditioner. Currently (in 2017), the worldwide installed RES capacity led by China accounts for more than 1500 gigawatt $^{1}$, as shown in Figure 1. Cheaper and easily manageable energy solutions attract more and more small-scale investors, suppliers and distributors, challenging the monopoly of traditional government electrical grids [1]. These off-grid systems not only produce energy but also reduce transmission losses and cost of production, distribution, and maintenance [2]. Work in [3] discusses a relay selection scheme for cellular networks, powered by green energy sources. However, the major goal is to reduce power consumption and dependency of cellular relay stations from traditional grids, the work is related to our proposed aggregation scheme for cellular network relays.

We propose smart meter based smart off-grid monitoring of RES using a number of sensors like moisture, light, motion, humidity, production, etc. and IoT middleware services/ servers. Installation of a number of smart meters, especially in urban areas requires communications network providers. Wi$\mathrm{Fi}$, Bluetooth, ZigBee, and other short-range communication technologies lack in range, internet, and prevalent service. On the other hand, the cellular networks have a widespread network with tons of base stations (BSs) having the range

\footnotetext{
${ }^{1}$ IRENA - Renewable Energy Capacity Statistics 2018, page 2-5
}

\section{LEADING COUNTRIES IN INSTALLED RENEWABLE ENERGY CAPACITY (GIGAWATT) IN 2017}

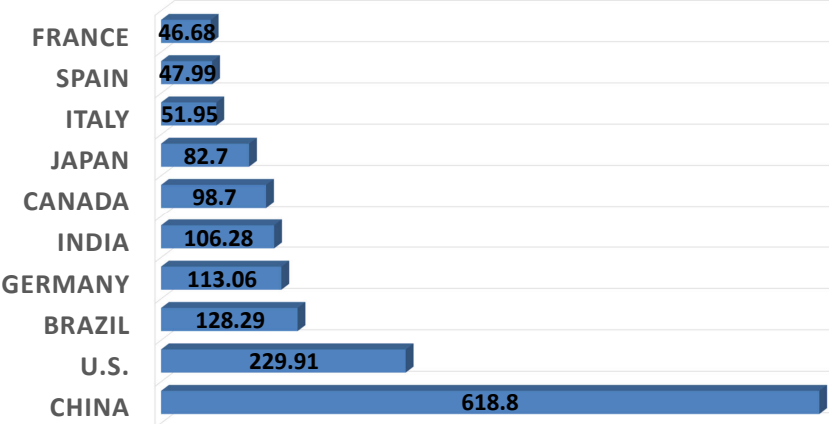

Fig. 1. Renewable energy capacity statistics

in kilometers and can be useful assets for the IoT communications. Moreover, the 3GPP standardized Narrow Band IoT (NB-IoT) with 4G Long Term Evolution (LTE) coverage characteristic with lower power consumption. However, the inherent random access channel (RACH) challenge persists. In NB-IoT communications, the device initializes the process after getting PRACH information from the SIB-2 message and transmits continuous repeated $\mathrm{RACH}$. The BS responds by sending RAR message and receives message 3 from the device. The BS sends contention resolution message to the device and initiates subsequent communications and resource assignments. In case of any message failure, the device resends preamble after $12 \mathrm{~ms}$. Unlike LTE, the RACH process messages are repeatedly sent between device and BS, illustrated in Figure 2. However, the collision or contention occurs similar to the LTE, if two or more than two devices send a request on the same randomly selected RACH. The contention burden is increased in the NB-IoT with repeated transmission occupying resources.

Authors in [4] describe that a device can withdraw its next scheduled transmission message if it gets unmatched Time Advance (TA) information of RAR and avoids possible request collision. However, there is still a lot of room to improve and reduce additional delays for the devices. Exhaustive study and observation of existing literature deduce that there is a need for an adequate and suitable architecture for smart RES management and control with continuous communications, which is not yet proposed. However, 5G network is embarking with a plethora of data rate to mobile devices but the delay intolerant IoT networks like smart grids and smart meters can make use of the $4 \mathrm{G}$ and NB IoT networks[5, 6]. On the 


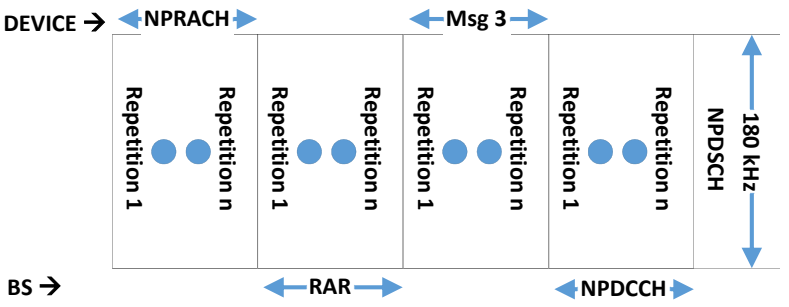

Fig. 2. NB IoT RACH procedure and structure

other hand, timely network connectivity becomes a critical need in a disaster situation [7]. We are the first to design and propose an outright architecture which not only discusses smart meter design and monitoring for RES but also tackles the communication challenges. Our major contribution and innovation over existing systems are:

1) design a smart meter and an innovative architecture for renewable sources

2) NB-IoT contention model with continuous monitoring of RES

3) higher signal-to-interference and noise (SINR) value by 20 times and reduced collisions and increase success by $25 \%$ over existing systems.

The rest of the article is organized as follows: Section 2 exhaustively discusses existing literature work on smart grids/ off-grid renewable systems and existing literature for the $4 \mathrm{G}$ LTE RACH and NB-IoT RACH issues.

Section 3 presents the proposed solution with smart meter design and proposed aggregation scheme. We analytically model SINR, collision and success probabilities with proof of concept. Performance evaluation and results are analyzed in Section 4. Section 5 concludes the paper.

\section{Literature REVIEW}

In this section, study of exhaustive literature is discussed, providing useful insights on what has been achieved and things that are lacking in earlier smart off-grid systems and 4G LTE RACH solutions.

\section{A. Smart Meter and Off-Grid}

There are numerous aspects in our take on the smart off-grid system like designing a smart meter for continuous monitoring and contributing with an intelligent and distributed architecture for control of RES. Existing literature is sporadically diverse in the areas like the design of micro-grids, distribution planning of RES, decentralize control, energy scheduling, etc.

Research work on small-scale RES in [1] focus on contractual trading of stored energy for conflicts avoidance using energy informatics. The authors model a coalitional game for direct trading among suppliers with fair revenue division. However, the trading requires a centralized entity as an aggregator in the main micro-grid station. Authors in [2] propose a microgrid design for RES and autonomous control overcharging or discharging of energy storage units. The proposed automation envisions to extract maximum power from the RES and provide quality power to the user. However, we plan to extend and provide deeper insights using a number of sensors and IoT middleware. Work in [3] discusses a relay selection scheme for cellular networks, powered by green energy sources. However, the major goal is to reduce power consumption and dependency of cellular relay stations from traditional grids, the work is related to our proposed aggregation scheme for cellular network relays. [8] introduce a multi-objective and multi-level model for the distribution system with RES and energy storage. The authors model RES and energy storage planning as an optimization problem using modified Pareto-based particle swarm optimization (PSO). Our proposed scheme can benefit [8] by providing pervasive and continuous monitoring and control. Another study in [9] model penetration of RES as multigenerator interconnected power network. The authors utilize a decentralized adaptive neural network feedback controller to stabilize dc link voltage oscillations during grid turbulence. The simulation and performance evaluation use IEEE 14bus power system for damping oscillations after disturbances. However, the study lacks in automation and in continuous control by the users.

Flexible supply-demand management in [10] focus on optimal energy scheduling for residential smart grids. Authors provide a solution for cost-effective energy scheduling with a centralized RES. The study highlights the trade-off between RES energy and associated cost, and volatility of RES for optimal exploitation. However, a major drawback of the study is to consider centralized renewable sources which question the practicality of the work. [11] utilizes a quality of experience (QoE) based approach for RES management in the residential environment. Authors propose profile based QoE aware appliance scheduling and RES power allocation. A central controller communicates with individual smart meters to change operational state on or off of the appliances. Authors in [12] assume a scenario of a number of RES in a centralized network and propose to balance RES in a micro-grid using meteorological forecast for next 24 hour and plant location. The solution depends on the weather forecast to enable most productive RES for energy generation. However, unlike [12], we consider communication challenges of continuous monitoring and insights of the RES using middleware [13].

\section{B. Random Access in LTE}

Inherently, NB-IoT utilizes Orthogonal Frequency Division Multiple Access (OFDMA) for resource sharing over RACH. In an OFDMA slot, each device randomly selects a RACH and in a case of multiple devices selecting the same RACH, a collision or contention happens [14]. Figure 2 outlines the repetitive access communication flow between device and BS. Initially, a device chooses a RACH and requests for resources. The BS responds with a random access response (RAR) message, containing a unique identifier. In the subsequent scheduled transmission, the BS realizes that two or more than two devices have been assigned the same RACH. The BS drops the requests and the devices are required to request again after a random back-off period. This adds delays in the communications resulting in user frustration and could cause catastrophic results in delay-intolerant applications like vehicular or medical. With the increase in the access intensity the chances of collision increase. 
TABLE I. LITERATURE REVIEW OF SMART RES AND RACH SOlutions

\begin{tabular}{|c|c|c|}
\hline Literature & Research Focus & Major Details \\
\hline \multicolumn{3}{|c|}{ Smart Off-Grid Solutions } \\
\hline Zhiyong Li et.al [1] & Small scale RES trading & $\begin{array}{l}\text { - Fair revenue and division model using coalitional game formulation } \\
\text { - Provides optimal consumption, prices and profit results }\end{array}$ \\
\hline S.K. Tiwari et.al [2] & Design and control of micro-grid & $\begin{array}{l}\text { - Autonomous RES control system } \\
\text { - Automatic charging and discharging }\end{array}$ \\
\hline Hui-Ju Hung et.al [3] & $\begin{array}{l}\text { Cellular relay selection with green en- } \\
\text { ergy }\end{array}$ & $\begin{array}{l}\text { - Relay selection } \\
\text { - Reduced power consumption of relay stations. } \\
\text { - Use of green energy for relays. }\end{array}$ \\
\hline Rui Li et.al [8] & Cooperative distribution system & $\begin{array}{l}\text { - RREs and energy storage planning and distribution } \\
\text { - Optimal solution using Pareto-based PSO }\end{array}$ \\
\hline $\begin{array}{l}\text { Shaghayegh Kazemlou et.al } \\
\text { [9] }\end{array}$ & Decentralized RES control & $\begin{array}{l}\text { - Decentralized controller for RESs and energy storage units } \\
\text { - Neural network controller }\end{array}$ \\
\hline Yuan $\mathrm{Wu}$ et.al [10] & Optimal energy scheduling & $\begin{array}{l}\text { - Cost effective and optimal exploited RES system } \\
\text { - Centralized RESs and energy storage units }\end{array}$ \\
\hline Virginia Pilloni et.al [11] & Smart home energy management & $\begin{array}{l}\text { - Smart meter based appliance energy controller } \\
\text { - Profile based approach for energy management }\end{array}$ \\
\hline Mattia Marinelli et.al [12] & Predictive control strategy for RES & $\begin{array}{l}\text { - Weather based forecast and RES control } \\
\text { - Day ahead energy planning }\end{array}$ \\
\hline Kab Seok Ko et.al [4] & Time alignment Matching & $\begin{array}{l}\text { ng RACH solutions } \\
\text { - Collision avoidance. } \\
\text { - Applicable only for overlapping area. }\end{array}$ \\
\hline Farhadi et.al[15] & Group based signaling (aggregation) & $\begin{array}{l}\text { - Device aggregation to reduce RACH competition } \\
\text { - Frequency reuse utilization. } \\
\text { - Possesses grouping overhead. }\end{array}$ \\
\hline Chang et.al [16] & Machine-to-Machine data gathering & $\begin{array}{l}\text { - A novel data perspective of Machine-to-Machine Communication. } \\
\text { - Incompatibility with large number of Machine-to-Machine. }\end{array}$ \\
\hline Zheng et.al [17] & $\begin{array}{l}\text { Prioritized Human and Machine Type } \\
\text { Communications }\end{array}$ & $\begin{array}{l}\text { - Extreme prioritization techniques } \\
\text { - Each techniques focuses only one type of communications } \\
\text { - Causes longer delays. }\end{array}$ \\
\hline Huasen et.al [18] & Interrupted Poisson Distribution & $\begin{array}{l}\text { - Active devices estimation in cellular system } \\
\text { - Device barring and congestion reduction. } \\
\text { - Causes longer delays. }\end{array}$ \\
\hline Shao-Yu Lien et.al [19] & Cooperative access barring & $\begin{array}{l}\text { - Barring parameter selection using Multiple eNB information } \\
\text { - Possesses less overhead. } \\
\text { - Applicable only for overlapping area. }\end{array}$ \\
\hline Tzu-Ming Lin et.al [20] & Dynamic ACB with device classification & $\begin{array}{l}\text { - Five categories for incoming traffic } \\
\text { - Dynamic access class barring utilization. } \\
\text { - Strategic, static approach. }\end{array}$ \\
\hline Hasan et.al [21] & Q learning at device end & $\begin{array}{l}\text { - Utilization of Q Learning in device } \\
\bullet \text { Collision avoidance. }\end{array}$ \\
\hline
\end{tabular}

Authors in [4] describe that a device can withdraw its next scheduled transmission message if it gets unmatched Time Advance (TA) information of RAR and avoids possible request collision. Work in [15] propose to reduce uplink requests by aggregating device requests. Initially, each device communicates to one another and selects a group delegate which corresponds to BS. Frequency reuse in the different group of devices increases the spectral efficiency within the same BS. However, the solution requires a great deal of frequency reuse and grouping management. Another similar work in [16] groups the devices and aggregate the uplink requests to reduce contention. The article models the size of the group as the NPHard problem and suggests two approaches, Cross Entropybased randomized approach, and Tabu Search. However, the solution is more focused on the group size problem than reducing RACH contentions. Another research in [17] evaluates two scenarios of Prioritized Human-Type-Communications and Prioritized Machine-Type-Communications to highlight the extreme communication procedures.

Authors of [18] discuss an Interrupted Poisson Distribution estimation approach for active user's calculation in the network. The number of active users, help in reducing $\mathrm{RACH}$ competition by device barring in the subsequent slot. On the other hand, the barred or delayed devices suffer from additional delays. [19] proposes a barring approach, where Access Class Barring (ACB) is improved to a cooperative design. Cooperative ACB gains $30 \%$ higher success over ACB, but a number of devices suffer from additional delays. Work in [20] outline five classes of incoming traffic and applies Dynamic Access Barring (DAB) mechanism to reduce RACH competition. The solution prioritizes the devices and adds delays to the traffic in three different scenarios of low, medium and high. [21] suggests Q-Learning experience based BS selection by the devices. The solution implicates devices in overlapping areas to have prior knowledge of BS. Standardization organizations like 3GPP have included ACB and Extended ACB mechanism to reduce collisions in LTE cellular networks [22]. However, there is still a lot of room to improve and reduce additional delays for the devices.

Table I outlines existing literature on state of the art solutions and ideas related to the smart grid and RESs. The table also includes existing solutions to tackle RACH congestion in communications resource access. Exhaustive study and observation of existing literature deduce that there is a need for an adequate and suitable architecture for smart RES management and control with continuous communications, which is not yet proposed. The strength of most of the existing solution is to enable a barring or prioritized system. We are the first to design and propose an outright architecture which not only discusses smart meter design and monitoring for RES but also tackles the communication challenges. 


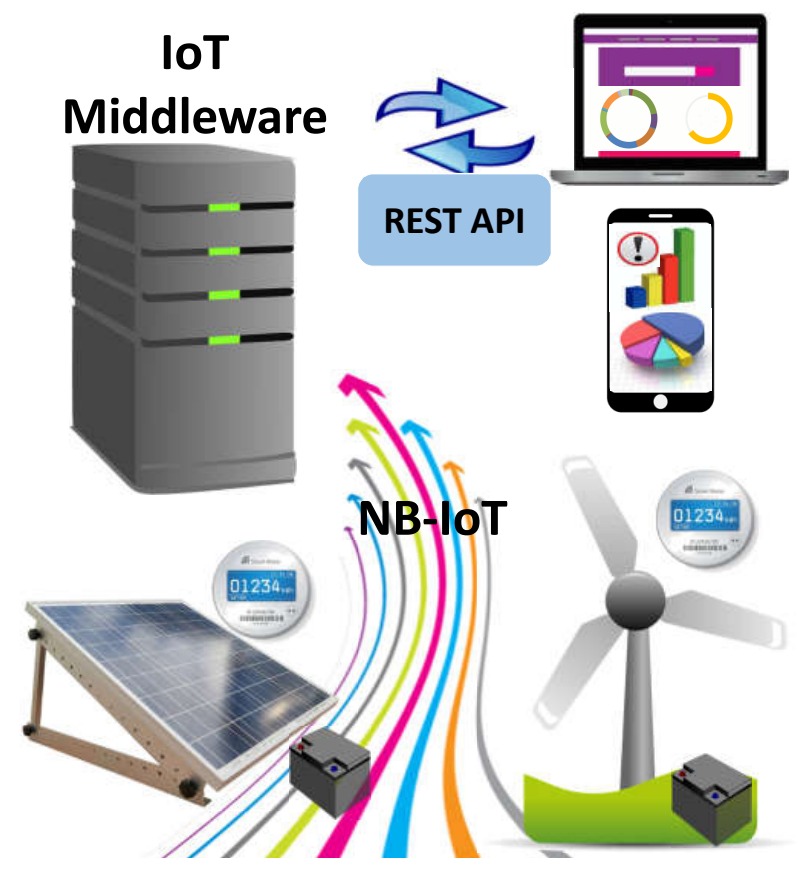

Fig. 3. Proposed paradigm of smart off-grid

\section{SMART OFF-GRID SOLUTION USING IOT}

Our design of smart meter considers a number of sensors like humidity, motion, etc. for continuous monitoring of the RES equipment. We propose that the information is then fed to a middleware service like ThingSpeak ${ }^{2}$, which not only provides data storage but also generates interactive graphs for deeper insights. Moreover, a mobile application or a web app can utilize ThingSpeak REST API to read the accessible and authorized data. Each communication message between a user and an RES equipment requires middleware server (ThingSpeak) and communications network. Figure 3 highlights middleware communications with the RES monitoring architecture over NB-IoT and 4G. The number of smart meters increases the access intensity in NB-IoT and 4G LTE RACH causing delays and the proposed scheme counters that by aggregating several smart meter requests. We propose that each device identifies itself as an aggregator using a boolean check variable Aggregator. If the device is an aggregator, it accepts the data, accumulates all messages and requests for RACH. On the contrary, the device broadcasts request to nearby devices, selects the first response of candidate aggregator and send data for accumulation.

A 4G BS shares resources using a number of random preambles which are accessed by the devices. Let $\mathrm{V}=\left\{v_{1}, v_{1}\right.$, $\left.\ldots, v_{k}\right\}$ be the total number of $k$ devices, cuncurrently requesting to the $4 \mathrm{G}$ BS for the $(M)$ RACH resources. Assuming that each of the devices compete with an equal opportunity, the collision probability $\left(P_{\alpha}\right)$ and success probability $\left(P_{\beta}\right)$ in the legacy network is desribed by [4] as:

$$
P_{\alpha}=1-\left(1-\frac{1}{M}\right)^{k-1}
$$

\footnotetext{
${ }^{2}$ ThingSpeak is the open IoT platform with MATLAB analytics. Online: [https://thingspeak.com/]
}

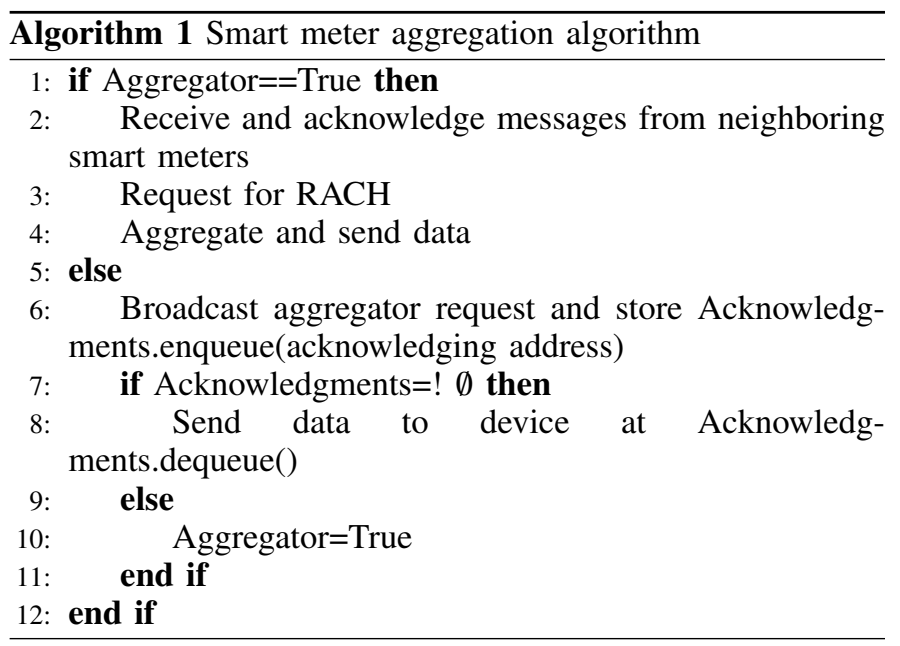

$$
P_{\beta}=\left(1-\frac{1}{M}\right)^{k-1}
$$

Moreover, the 4G LTE uplink Signal-Interference-plusNoise-Ratio (SINR) $\left(\delta_{\alpha}\right)$ of a smart meter with $k$ other devices with $P$ transmission power, $g$ channel gain, and $\mu$ Additive White Gaussian Noise off a quasi-static Rayleigh fading channel, is described in [5] as:

$$
\delta_{\alpha}=\frac{g P}{\mu+\sum_{n=1}^{k-1} g_{n} P_{n}},
$$

where interference by other $k$ devices is represented by $\sum_{n=1}^{k-1} g_{n} P_{n}$.

The data rate $\left(\psi_{\alpha}\right)$ with $B W$ bandwidth of $4 \mathrm{G}$ network, can be estimated as:

$$
\psi_{\alpha}=B W \log \left(1+\delta_{\alpha}\right)
$$

Let $R_{B}$ be the coverage radius of a $\mathrm{BS}$ and $R_{S}$ be the range of an aggregating smart meter. If another smart meter is randomly placed in the radius of the BS then the probability of its placement within the range of an aggregator can be calculated as $P_{\rho}=\frac{\pi R_{S}^{2}}{\pi R_{B}{ }^{2}}$. We can extend the probability for randomly placed $\lambda$ aggregators, as:

$$
P_{\rho}=\frac{\sum_{a=1}^{\lambda} \pi R_{S, a^{2}}-\left[\left(\sum_{b=1}^{\lambda} \sum_{c=1}^{\lambda} \pi R_{S, b^{2}}{ }^{2} \cap \pi R_{S, c^{2}}\right) / 2\right]}{\pi R_{B}{ }^{2}}
$$

Because, the aggregators are randomly placed, they can overlap each other. Above equation first calculates total radius of all $\lambda$ aggregators as $\sum_{a=1}^{\lambda} \pi R_{S, a^{2}}$. Subsequently, the overlapping radius points are substracted using second part, $\left[\left(\sum_{b=1}^{\lambda} \sum_{c=1}^{\lambda} \pi R_{S, b}{ }^{2} \cap \pi R_{S, c}{ }^{2}\right) / 2\right]$. Combining both parts give us total favourable outcomes which then divided by total possible outcomes $\pi R_{B}{ }^{2}$ estimates probability of a randomly 
placed smart meter within the range of an aggregator. However, for better understanding and simplicity of complex equation, we assume that the aggregators are disjoint to each other, which makes second term of above equation $\left[\left(\sum_{b=1}^{\lambda} \sum_{c=1}^{\lambda} \pi R_{S, b}{ }^{2} \cap \pi R_{S, c}{ }^{2}\right) / 2\right]$ equal to 0 . Moreover, it also puts a constraint that to total radius points of $\lambda$ aggregators must not exceed the total possible outcomes, i.e. $\sum_{a=1}^{\lambda} \pi R_{S, a^{2}}<$ $\pi R_{B}^{2}$.

Considering that there are $k$ devices randomly placed within a BS, the total number of devices in $\lambda$ aggregators can be estimated as:

$$
\rho=\frac{\sum_{a=1}^{\lambda} \pi R_{S, a^{2}}}{\pi R_{B}^{2}} \times k+\lambda
$$

Considering that the proposed aggregation reduces the access intensity by $\rho$ devices, the collision probability $\left(P_{\gamma}\right)$ and success probability $\left(P_{\omega}\right)$ in the proposed architecture can be estimated as:

$$
\begin{gathered}
P_{\gamma}=1-\left(1-\frac{1}{M}\right)^{k-1-\rho} \\
P_{\omega}=\left(1-\frac{1}{M}\right)^{k-1-\rho}
\end{gathered}
$$

Moreover, the reduced competition also reduces the interference in the network, ergo the SINR in the proposed system $\left(\delta_{\beta}\right)$ becomes:

$$
\delta_{\beta}=\frac{g P}{\mu+\sum_{n=1}^{k-1-\rho} g_{n} P_{n}},
$$

Subsequently, better $\operatorname{SINR}\left(\delta_{\beta}\right)$ increases the data rate $\left(\psi_{\beta}\right)$ for a device in the proposed paradigm with same $B W$ bandwidth. Mathematically:

$$
\psi_{\beta}=B W \log \left(1+\delta_{\beta}\right)
$$

The proposed system provides lower collision and higher successful access to the devices in the presence of smart meters. Our claim stands if following hypothesis holds true: $X=\frac{\text { Proposed Collision Probability }}{\text { Existing Collision Probability }}=\frac{P_{\gamma}}{P_{\alpha}}>1$. Replacing values of $P_{\gamma}$ from Equation 7 and $P_{\alpha}$ from Equation 1 in the equation, gives:

$$
\left[X=\frac{1-\left(1-\frac{1}{M}\right)^{k-1-\rho}}{1-\left(1-\frac{1}{M}\right)^{k-1}}\right]>1
$$

\begin{tabular}{|c|c|c|}
\hline Symbol & Description & $\begin{array}{l}\text { Simulation } \\
\text { Value }\end{array}$ \\
\hline $\mathrm{k}$ & Number of users & $25-250$ \\
\hline $\mathrm{M}$ & $\begin{array}{llll}\begin{array}{l}\text { Number } \\
\text { (RACH) }\end{array} & \text { of } & \text { available } & \text { resources } \\
\end{array}$ & 64 \\
\hline BW & 4G LTE, NB-IoT & $\begin{array}{l}20 \mathrm{MHz}, \quad 180 \\
\mathrm{KHz}\end{array}$ \\
\hline$\rho$ & Number of aggregated users & N/A \\
\hline$\lambda$ & Number of aggregator & $4-20$ \\
\hline$P_{\alpha}$ & $\begin{array}{l}\text { Collision probability in existing sys- } \\
\text { tem }\end{array}$ & N/A \\
\hline$P_{\beta}$ & $\begin{array}{l}\text { Collision probability in proposed sys- } \\
\text { tem }\end{array}$ & N/A \\
\hline$P_{\gamma}$ & Success probability in existing system & N/A \\
\hline$P_{\omega}$ & $\begin{array}{l}\text { Success probability in proposed sys- } \\
\text { tem }\end{array}$ & N/A \\
\hline$\delta_{\alpha}$ & SINR in the existing system & N/A \\
\hline$\delta_{\beta}$ & SINR in the proposed system & N/A \\
\hline$\psi_{\alpha}$ & data rate in the existing system & N/A \\
\hline$\psi_{\beta}$ & data rate in the proposed system & N/A \\
\hline$P$ & Transmission power & $250 \mathrm{~mW}$ \\
\hline$g$ & Channel gain & $15 \mathrm{dBi}$ \\
\hline$\mu$ & Additive White Gaussian Noise & $-101 \mathrm{dBm}$ \\
\hline$R_{B}$ & Range of $4 \mathrm{G}$ base station (BS) & $500 m$ \\
\hline$R_{S}$ & Range of smart meter aggregator & $50 m$ \\
\hline
\end{tabular}

Applying $\log [X]$ and reducing:

$$
\left[\begin{array}{l}
\log [X]=(k-1-\rho) \times \log \left[1-\left(1-\frac{1}{M}\right)\right]- \\
(k-1) \times \log \left[1-\left(1-\frac{1}{M}\right)\right]
\end{array}\right]>0
$$

TABLE II. Symbols AND SimUlation PARAMETERS

$$
\begin{gathered}
{\left[\begin{array}{l}
\log [X]=\log \left[1-\left(1-\frac{1}{M}\right)\right] \times \\
{[(k-1-\rho)-(k-1)]}
\end{array}\right]>0} \\
{\left[\log [X]=\log \left[1-\left(1-\frac{1}{M}\right)\right] \times-\rho\right]>0}
\end{gathered}
$$

The term in above equation $\left(\log \left[1-\left(1-\frac{1}{M}\right)\right] \times-\rho\right)$ will always provide a positive value where $\rho>1$ and $M>1$. It should be noted that in every possible scenario, a BS will have more than one resources $(M)$ and more than one aggregations $(\rho)$. Thus proving $\log [X]>0 \Rightarrow X>1 \Rightarrow P_{\gamma}>P_{\alpha}$, where $\rho>1$ and $M>1$. It is safe to assume that a similar hypothesis also holds true for success probability and SINR values.

\section{PERFormance EVAlUation}

Our Monte-Carlo simulation based experiments and results for the existing and proposed system includes a total of 25 to 250 devices and $64 \mathrm{RACH}$ preambles (M) for $20 \mathrm{MHz}$ (LTE) and $180 \mathrm{KHz}$ (NB-IoT) bandwidth (BW) based 4G LTE BS with $500 \mathrm{~m}$ communications radius [23]. The number of aggregators is defined between 4 to 20 , having $50 \mathrm{~m}$ radius each, outlined in Table II. Each device/ smart meter is programmed to have a $250 \mathrm{~mW}$ transmission power, 15 $\mathrm{dBi}$ channel gain and $-101 \mathrm{dBm}$ noise factor. Comparative analysis is carried out using existing legacy system benchmarks presented in [4] and [5]. The existing legacy models are implemented with similar parameters and on similar MonteCarlo simulation settings.

The increase in the number of requests and devices impacts the network performance. Assume a scenario of crowded urban environment where people are almost continuously communicating through mobile devices. Thus, increasing the accessing intensity and RACH collision which result in packet drops and additional delays. These delays become critical in delay intolerant applications. Our system aggregates the requests automatically and reduces the access intensity without barring any device. Figure 4 shows that the proposed smart 


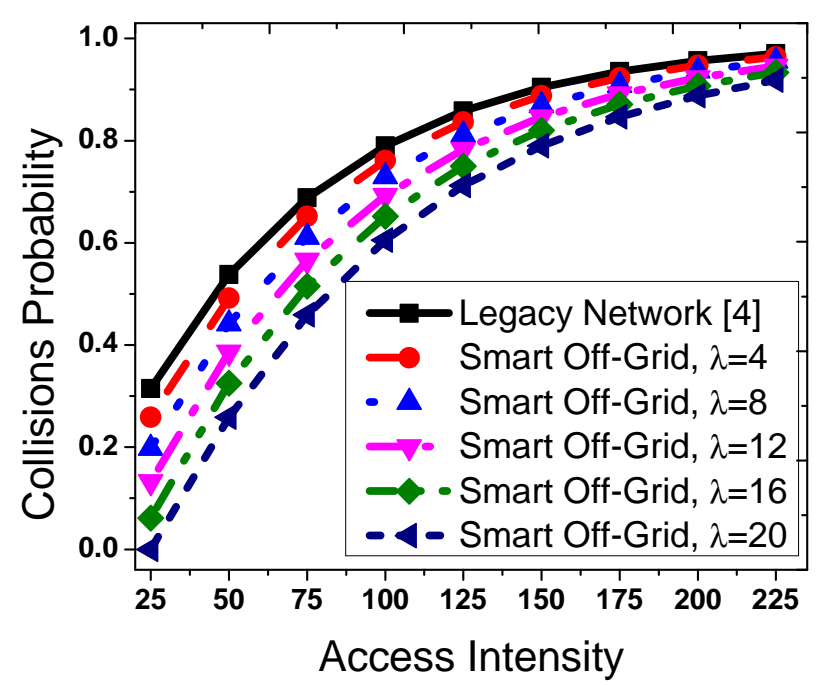

Fig. 4. Collision probability in the proposed and legacy system

off-grid system reduces collision probability by $5 \%$ to $25 \%$ for 4 to 20 aggregators. The existing collision probability is calculated using Equation 1 and proposed collision probability is estimated using Equation 7. The legacy network suffers from higher collisions due to the increase in the number of devices, whereas, the proposed smart off-grid reduces access intensity by aggregating requests. However, the increase in access intensity equally impacts both systems and increases collisions.

Figure 5 illustrates that the proposed system provides access to all devices at access intensity equals 25 . The results for existing and proposed success probabilities are estimated using Equation 2 and 8. The successful resource allocation reduces in the proposed scheme with the increase in the number of devices. However, the proposed scheme outperforms the legacy system by $25 \%$ success probability. Figure 6 highlights the SINR gain of the proposed scheme over the existing network using Equation 3 and 9. The number of aggregators $(\lambda)$ has a huge impact on the SINR value. The proposed scheme with 4 to 20 aggregators achieves approximately $1.3 \times$ to $20 \times$ gain over the legacy system. Figure 7 shows that the increase in devices equally impact on legacy network and proposed scheme with all variations. With a bandwidth of $20 \mathrm{MHz}$ BW and only $\lambda=4$, the proposed scheme outperforms the legacy system by $\sim 2$ Mbps. The increase in the value of $\lambda$ increase the performances of the proposed scheme, i.e. with $\lambda=20$, the data rate soars high as $43.97 \mathrm{Mbps}$. The channel quality indicator (CQI) of devices impact bandwidth distribution, ergo reducing data rate per device. Considering NB-IoT limited bandwidth of $180 \mathrm{KHz}$, we have experimented the data rate for 25 to 250 devices, competing against each other. NB-IoT specific evaluation in Figure 8 also present similar facts that the proposed scheme outperforms the legacy system by providing three times higher value.

\section{A. Complexity Analysis}

The time and order complexity of proposed aggregation algorithm in a best-case scenario includes that the device is aggregator and shares only one message. In response, receives

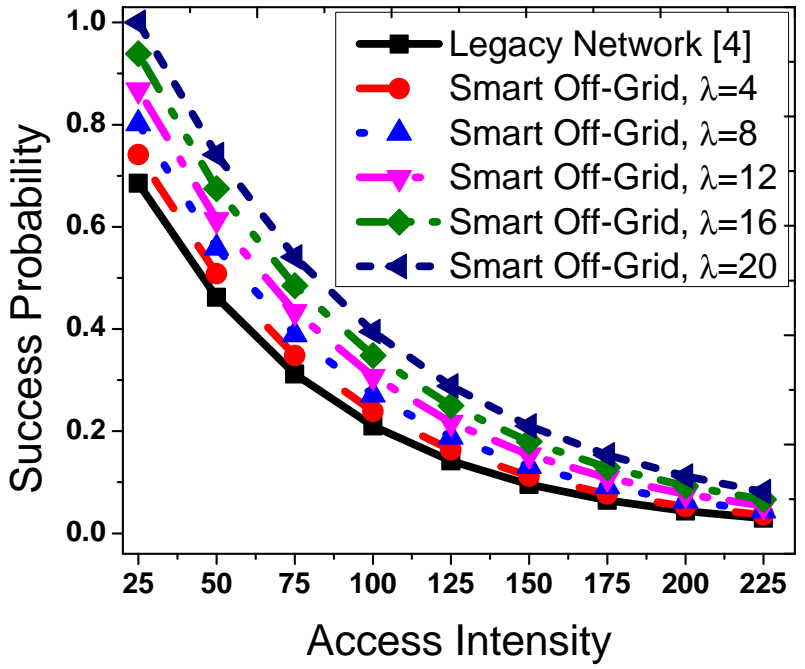

Fig. 5. Success probability in the proposed and legacy system

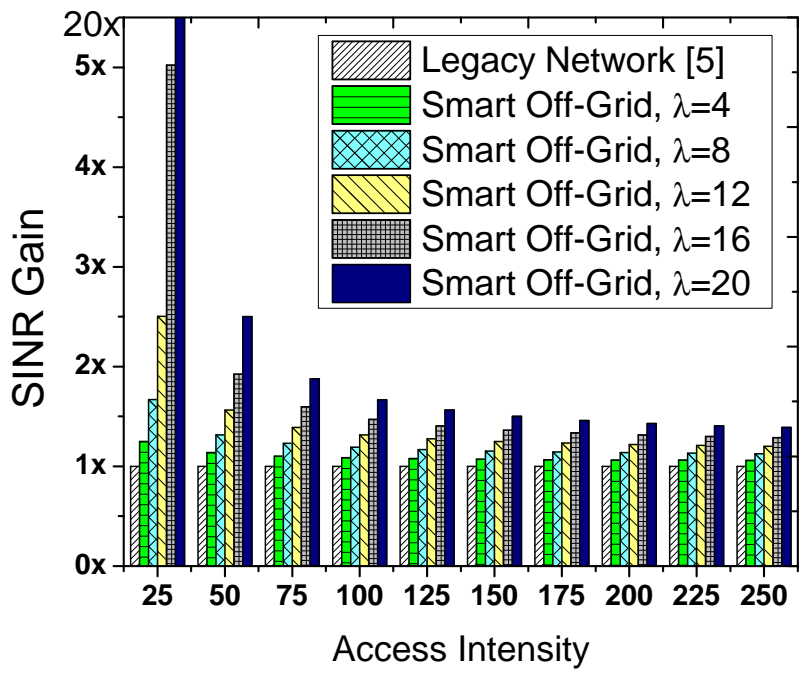

Fig. 6. SINR gain in the proposed and legacy system

neighboring messages and request for RACH. Thus, using Big$\mathrm{O}$ notation, the total complexity of the best case scenario is $O(1)+O(N)+O(1)$. On the other hand, if the device is not an aggregator, it requests to all neighboring devices $(O(N))$ and receives all possible acknowledgment, but chooses the first response. Nevertheless, the total complexity becomes $O(N)+$ $O(N)+O(1)$. In both cases, the number of devices plays a major role to increase complexity but this number also increase the chances of successful aggregation. Thus, the message exchange becomes acceptable for all those devices requiring a successful connection and communications.

\section{CONClusion}

This article presents and provides a complete architecture and communications paradigm to keep track of distributed RES equipment. Our major contributions include exhaustive literature review and innovative solution. We propose to continuously keep track of the performance and efficiency of the RES equipment using a smart meter. Our innovative smart meter not only tracks the energy generation but also monitors the 


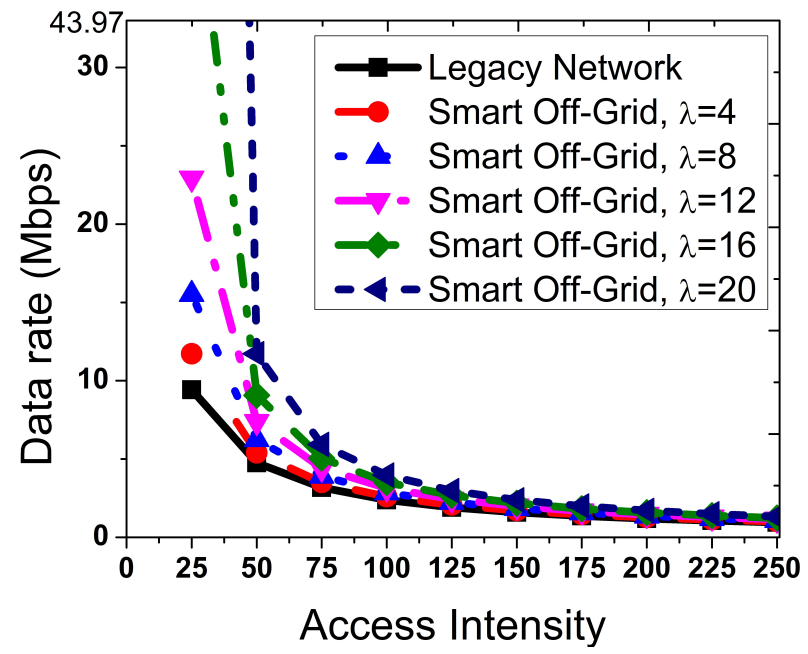

Fig. 7. Data rate of $4 \mathrm{G}$ LTE in the proposed and legacy system

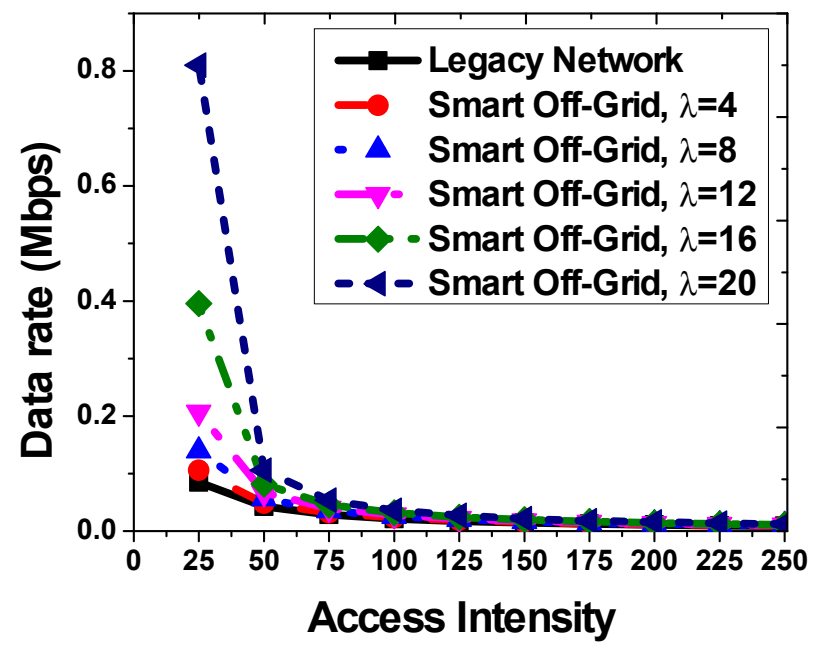

Fig. 8. Data rate of NB-IoT in the proposed and legacy system

health of the equipment. Moreover, the communication model for pervasive communication over NB-IoT is modeled and an aggregation scheme is presented. Our analytical model and respective proof of concept corroborate our claims of pervasive communications over $4 \mathrm{G}$ LTE and show clear advantages over the existing systems. Smart meter aggregation in NBIoT and $4 \mathrm{G}$ environment achieve $25 \%$ reduced collisions and increased success probability. The communication also gains 1.3 to 20 times higher SINR value and three times higher data rate for a device (in LTE and NB-IoT, both), which leads to uninterrupted access. Our work and mathematically tractable equations pave a bridge to the co-existence of the NB-IoT in LTE and future 5G communications network. Moreover, in a device-centric architecture the trustworthy cooperation and aggregation of the devices is also a major future work.

\section{REFERENCES}

[1] Zhiyong Li, Lin Chen, and Guofang Nan: 'Small-Scale Renewable Energy Source Trading: A Contract Theory Approach', IEEE Transcation on Industrial Informatics, vol. 14, no. 4, pp. 1491-1500, 2018

[2] S.K. Tiwari, Bhim Singh, and P.K. Goel, 'Design and Control of MicroGrid fed by Renewable Energy Generating Sources', IEEE Trans. on Industry Applications, vol. 54, no. 3, pp. 2041-2050, 2018.
[3] Hui-Ju Hung, Ting-Yu Ho, Shi-Yong Lee, Chun-Yuan Yang, and DeNian Yang, 'Relay Selection for Heterogeneous Cellular Networks with Renewable Green Energy Sources', IEEE Trans. on Mobile Computing, vol. 17, no. 30, pp. 1-14, 2018.

[4] K.S. Ko, M.J. Kim, K.Y. Bae, D.K. Sung, J.H. Kim, and J.Y. Ahn, 'A novel random access for fixed-location machine-to-machine communications in OFDMA based systems'. Communications Letters, IEEE, vol. 16, no. 9, pp. 1428-1431, 2012.

[5] F. H. Kumbhar, N. Saxena, A. Roy, 'Reliable Relay Autonomous Social D2D Paradigm for 5G LoS Communications'. IEEE Communications Letters, vol. 21, no. 7, pp. 1593-1596, 2017.

[6] N. Jiang, Y. Deng, M. Condoluci, W. Guo, A. Nallanathan and M. Dohler, 'RACH Preamble Repetition in NB-IoT Network'. IEEE Communications Letters, vol. 22, no. 6, pp. 1244-1247, 2018.

[7] N. Saxena, M. Agiwal, H. Ahmad and A. Roy, 'D2D-based Survival on Sharing (SoS) for Enhanced Disaster Time Connectivity', IEEE Technology and Society Magazine, vol.37, no. 3, pp. 64-73, Sept. 2018.

[8] Rui Li, Wei Wang, and Mingchao Xia, "Cooperative Planning of Active Distribution System With Renewable Energy Sources and Energy Storage Systems", IEEE Access, vol. 6, pp. 5916-5926, 2017.

[9] Shaghayegh Kazemlou, and Shahab Mehraeen, "Novel Decentralized Control of Power Systems With Penetration of Renewable Energy Sources in Small-Scale Power Systems", IEEE Transactions on Energy Conversion, vol. 29, no. 4, pp. 851-861, 2014.

[10] Yuan Wu, Vincent K. N. Lau, Danny H. K. Tsang, Li Ping Qian, and Limin Meng, "Optimal Energy Scheduling for Residential Smart Grid with Centralized Renewable Energy Source", IEEE Systems Journal, vol. 8, no. 2, pp. 562-576, 2014.

[11] Virginia Pilloni, Alessandro Floris, Alessio Meloni, and Luigi Atzori, "Smart Home Energy Management Including Renewable Sources: A QoEdriven Approach", IEEE Transactions on Smart Grid, vol. 9, no. 3, pp. 2006-2018, 2016.

[12] Mattia Marinelli, Fabrizio Sossan, Giuseppe Tommaso Costanzo, and Henrik W. Bindner, "Testing of a Predictive Control Strategy for Balancing Renewable Sources in a Microgrid", IEEE Transactions on Sustainable Energy, vol. 5, no. 4, pp. 1426-1433, 2014.

[13] C. Perera, C.H. Liu, S. Jayawardena, and M. Chen, "A survey on internet of things from industrial market perspective", IEEE Access, vol. 2, pp. 1660-1679, 2014.

[14] L. M. Bello, P. D. Mitchell and D. Grace, 'Intelligent RACH Access Techniques to Support M2M Traffic in Cellular Networks,' in IEEE Transactions on Vehicular Technology, vol. 67, no. 9, pp. 8905-8918, Sept. 2018

[15] G.Farhadi, and A.Ito, "Group-based signaling and access control for cellular machine-to-machine communication", In IEEE Vehicular Technology Conference (VTC Fall), IEEE 78th, 2013 (pp. 1-6), 2013

[16] C.H. Chang, and H.Y. Hsieh, "Not every bit counts: A resource allocation problem for data gathering in machine-to-machine communications". In IEEE Global Communications Conference (GLOBECOM), pp. 5537-5543, 2012.

[17] K. Zheng, S. Ou, J. Alonso-Zarate, M. Dohler, F. Liu, and H. Zhu, "Challenges of massive access in highly dense LTE-advanced networks with machine-to-machine communications", Wireless Communications, IEEE, vol. 21, no. 3, pp. 12-18, 2014.

[18] H.Wu, C. Zhu, R.J. La, X.Liu, and Y. Zhang, FASA, “ Accelerated S-ALOHA using access history for event-driven M2M communications, Networking, IEEE/ACM Transactions on, vol. 21, no. 6, pp. 1904-1917, 2013

[19] S.Y Lien, T.H. Liau, C.Y. Kao, and K.C Chen, "Cooperative access class barring for machine-to-machine communications". Wireless Communications, IEEE Transactions on, vol. 11, no. 1, pp. 27-32, 2012.

[20] T. M. Lin, C. H. Lee, J. P. Cheng and W. T. Chen, "PRADA: Prioritized Random Access With Dynamic Access Barring for MTC in 3GPP LTE-A Networks," IEEE Transactions on Vehicular Technology, vol. 63, no. 5, pp. 2467-2472, Jun 2014.

[21] M. Hasan, E. Hossain, and D. Niyato, "Random access for machineto-machine communication in LTE-advanced networks: issues and approaches". Communications Magazine, IEEE, vol. 51, no. 6, pp. 86-93, 2013.

[22] 3GPP. Technical Specification 37.868, Study on RAN Improvements for Machine-type Communications. 2011, URL: www.qtc.jp/3GPP/Specs/37868-b00.pdf [Access Date: 5 July 2017]

[23] F. H. Kumbhar, A. Roy and N. Saxena, "RoBiN: Random Access using Border Routers in Cellular Netw.", Mobile Netw. and Applications, vol. 21, no. 4, pp. 620-634, 2016. 